\title{
Sistema de gestión de la calidad y expectativas de desarrollo en docentes de una universidad privada
}

\author{
Quality management system and development expectations in teachers of a private university
}

Sistema de gestão da qualidade e expectativas de desenvolvimento em professores de uma universidade privada

\section{ARTÍCULO DE INVESTIGACIÓN}

Ariadna Hernández Castillo

ariadnahernandezdetejeda@gmail.com

https://orcid.org/0000-0002-2964-2782

\author{
Ofelia Carmen Santos Jiménez \\ ofelia.santos@unmsm.edu.pe \\ https://orcid.org/0000-0003-1294-0641
}

Ada Gallegos Ruiz Conejo

agallegosr@unmsm.edu.pe

https://orcid.org/0000-0002-8264-711X

\author{
Alejandra Duvina Romero Díaz \\ aromerod@usmp.pe \\ https://orcid.org/0000-0002-8117-2319
}

Universidad Nacional Mayor de San Marcos, UPG, Lima-Perú

Recibido 28 de abril 2021 | Arbitrado y aceptado 25 de mayo 2021 | Publicado en 01 julio 2021

\section{RESUMEN}

El artículo analiza los criterios de calidad que se vienen gestionando, el objetivo fue determinar la relación entre el sistema de gestión de la calidad y las expectativas de desarrollo en docentes de una universidad privada. La investigación es descriptivo-correlacional, aplicativa. Muestra no probabilística conformada por 465 docentes. Para recopilar datos se aplicó la técnica de encuesta, se utilizó dos instrumentos: escala sobre sistema de gestión de calidad (SIGECA) y escala de desarrollo docente (EXDO), validada por juicio de expertos. Los estadísticos empleados: Rho de Spearman, Kolmogórov-Smirnov y Shapiro-Wilk. Los resultados muestran que las implicancias son discutidas en función de la realidad sociolaboral del país. Concluye que existe relación directa .781, entre sistema de gestión de calidad y expectativas de desarrollo del docente.

Palabras clave: Sistema de gestión de calidad; Satisfacción laboral; Expectativa de desarrollo docente; organización institucional, Mejora continua

\section{ABSTRACT}

The article analyzes the quality criteria that are being managed, the objective was to determine the relationship between the quality management system and the development expectations in teachers of a private university. The research is descriptive-correlational, applicative. Non-probabilistic sample made up of 465 teachers. To collect data, the survey technique was applied, two instruments were used: scale on quality management system (SIGECA) and scale of teacher development (EXDO), validated by expert judgment. The statisticians used: Rho de Spearman, KolmogórovSmirnov and Shapiro-Wilk. The results show that the implications are discussed according to the socio-labor reality of the country. It concludes that there is a direct relationship .781 between the quality management system and teacher development expectations.

Key words: Quality management system; Job satisfaction; Expectation of teacher development; Institutional organization, continuous improvement

\section{RESUMO}

$\mathrm{O}$ artigo analisa os critérios de qualidade que estão sendo administrados, o objetivo era determinar a relação entre o sistema de gestão da qualidade e as expectativas de desenvolvimento dos professores em uma universidade privada. A pesquisa é descritivo-correlacional, aplicada. A amostra não-probabilística consistia de 465 professores. A técnica de pesquisa foi utilizada para coletar dados, e dois instrumentos foram utilizados: a escala do sistema de gestão da qualidade (SIGECA) e a escala de desenvolvimento de professores (EXDO), validada por julgamento de especialistas. As estatísticas utilizadas foram Spearman's Rho, Kolmogorov-Smirnov e ShapiroWilk. Os resultados mostram que as implicações são discutidas de acordo com a realidade sócio-laboral do país. Ela conclui que existe uma relação direta .781, entre o sistema de gestão da qualidade $\mathrm{e}$ as expectativas de desenvolvimento dos professores.

Palavras-chave: Sistema de gestão de qualidade; Satisfação no trabalho; Expectativa de desenvolvimento dos professores; Organização institucional, Melhoria contínua 


\section{INTRODUCCIÓN}

Gestionar una universidad como la Universidad Privada del Norte (en adelante UPN), miembro de Laureate International Universities, exige contar con directivos, con mucho liderazgo e integridad y que siga la misión de Becker, D., fundador de Laureate Education Inc.: "Laureate es una comunidad internacional de universidades que fomenta el aprendizaje sin fronteras. El propósito es ofrecer educación superior con una perspectiva multicultural única, y promover profesionales para que desarrollen carreras apasionantes y obtengan logros a lo largo de su vida, que triunfen y sirvan de beneficio a la sociedad.

Bajo esta misión se insertala propuesta educativa de esta universidad: "Formar ciudadanos dueños de su propio destino que aporten positivamente a la sociedad". La Red Laureate Education Inc. (en adelante LIU) y UPN tienen la convicción, que la educación puede cambiar vidas.

En ese sentido el rol del docente es fundamental, no obstante que para que el docente actúe, es necesario que desde la organización (UPN) se establezca con claridad las líneas maestras de dirección. Por ello, es relevante que se re-conozca la calidad como parte de la cultura organizacional.

La UPN ha declarado públicamente su política de calidad. Los criterios de dicha calidad, como: la calidad educativa y organizacional, que se orienta en la búsqueda de la satisfacción de los estudiantes, los empleados y la comunidad en general, brindando servicios educativos enfocados en el logro de competencias y centrados en el estudiante; vinculando el quehacer académico al desarrollo de actividades de investigación y proyección social. El cumplimiento de los requisitos legales vigentes y otros requisitos aplicables a sus servicios, que la universidad suscriba. Para cumplir con estas políticas, la UPN cuenta con el Vicerrectorado de Calidad Educativa, la Dirección de Planificación y Mejora Continua y la Gerencia de Procesos, que se encargan de consolidar, sostener y desarrollar el sistema de gestión de calidad de la universidad.

Por ello, lo que se proponga organizativamente debe adecuarse en el aula y perfeccionarse desde ahí. Es en el espacio áulico donde se vive la "verdad" y es ahí donde el docente junto al estudiante más el soporte curricular, se convierten en protagonistas de todo lo que la organización trabaja para cumplir con el perfil del egresado que ofrece a la sociedad y es el docente quien finalmente lleva toda la estrategia al estudiante, por lo tanto, es con él con quien se debe trabajar de la mano para que la misión y visión se cumpla. Por tanto, es pertinente articular la gestión de la calidad y lo que necesitan los docentes de esta universidad.

Es así que el Sistema de Gestión de la Calidad y expectativas de desarrollo en los docentes de una universidad privada del país 2018 que se gesta en el Vicerrectorado de Calidad Educativa, a donde pertenece la Dirección de Desarrollo Docente, junto con la Dirección Nacional de Calidad Educativa, la Dirección de Planificación y Mejora Continua y la Gerencia de Procesos. Es un proyecto con un enfoque prospectivo, como respuesta a las tendencias que enfrenta la sociedad peruana, determinada por los cambios que se producen a nivel mundial en los diversos ámbitos de la actividad humana. (CEPLAN, 2010).

\section{Sistema de gestión de calidad educativa}

El SINEACE (2018), define como un conjunto de secciones que constituyen la organización de programas académicos que interactúan 
estableciendo políticas, objetivos y procesos que permiten alcanzar las metas institucionales vinculadas con la gestión de calidad. La norma ISO 21001:2018, establece los requisitos para el cumplimiento del sistema de gestión de las organizaciones educativas. Ello permite establecer el mapa de procesos, de tal manera que se visualice el funcionamiento y desempeño, con toda claridad para el desarrollo del plan estratégico. Este estudio promueve que la UPN debe prepararse para el mediano y largo plazo, y ello pasa entre otras acciones por generar, fortalecer y mantener la línea de sucesión de sus cuadros de línea (docentes), que harán posible la implementación del sistema de gestión de calidad.

En cuanto al sistema de gestión de la calidad de la educación, la UPN redactó la Resolución de Rector No. 004-2016-UPN, decidiendo aprobar la constitución del Comité de Calidad de la UPN, cuya función general es aprobar el plan de trabajo general para implementar, mantener y desarrollar la calidad de la UPN. Apoyar y asistir en el proceso de implementación de SGC en UPN. Establecer un mecanismo que permita a diferentes procesos aplicar políticas de calidad. Aprobar el plan de auditoría de calidad y evaluar sus resultados. Comunicar y promover a la comunidad UPN sobre la política de calidad aprobada por la alta dirección. Establecer grupos de trabajo para mejorar los procesos académicos. Informe mensual a la alta dirección de la UPN (comité ejecutivo) sobre la marcha del proyecto "Sistema de gestión de la calidad basado en el modelo 150 9000".

Sobre el Sistema de Gestión de Calidad Educativa, la UPN elaboró su Resolución Rectoral $\mathrm{N}^{\circ}$ 004-2016-UPN, donde resuelven aprobar la creación del Comité de Calidad UPN, cuyas funciones generales, son: aprobar el Plan general de Trabajo para la implementar, sostener y desarrollar el Sistema de Gestión de Calidad de UPN. Apoyar y asistir el proceso de implementación del SGC en UPN. Establecer los mecanismos que permitan a los diferentes procesos la aplicación de políticas de calidad. Aprobar programas de auditorías de calidad y evaluar sus resultados. Comunicar y sensibilizar a la comunidad UPN sobre las políticas de calidad aprobadas por la alta dirección. Instalar grupos de trabajo para la mejora de los procesos académicos. Informar mensualmente a la Alta Dirección de UPN (Comité Ejecutivo) sobre el avance del proyecto "Sistema de gestión de calidad basado en el modelo 150 9000".

En 2017, formuló una política de calidad, manifestando que la calidad es parte de su cultura organizacional, por lo que prometieron: Educación y calidad organizacional, orientada a buscar la satisfacción de estudiantes, empleadores y toda la comunidad, y brindar servicios educativos clave centrados en la logro de la competencia, centrado en el estudiante; vinculando el trabajo académico con el desarrollo de actividades de investigación y proyecciones sociales. Para cumplir con los requisitos legales vigentes y otros requisitos que se aplican a nuestros servicios, la universidad se suscribe a estos requisitos.

Para cumplir con esta política, la UPN cuenta con la Dirección Nacional de Calidad Educativa, la Dirección de Planificación y Mejora Continua y la Gerencia de Procesos, encargado de consolidar, sostener y desarrollar el Sistema de Gestión de Calidad de la Universidad. Se plantean sus objetivos basados en la calidad de la educación y organización 
tiene como objetivo buscar la satisfacción de estudiantes, empleadores y toda la comunidad; brindar servicios educativos enfocados en el logro de habilidades y centrados en el estudiante; vincular el trabajo académico con el desarrollo de actividades de investigación y proyecciones sociales. Para cumplir con los requisitos legales vigentes y otros requisitos que se aplican a los servicios de la universidad se suscribe a estos requisitos.

En materia de certificación, SINEACE (2017) es de carácter público, y su finalidad es certificar sus proyectos de aprendizaje de acuerdo con los estándares de calidad especificados en la "Modalidad de Certificación de Proyectos de Aprendizaje de Educación Superior Universitaria". UPN cree que este es un proceso mediante el cual un organismo de certificación reconoce oficialmente que una carrera cumple con los estándares de calidad previamente establecidos y la trata como una oportunidad para que otras carreras y toda la organización mantengan un ciclo de mejora continua. Para la UPN, la certificación es: asegurar que un tercero autorizado verifique y apoye a la universidad para asegurar la calidad de sus estudiantes, satisfaciendo así a la sociedad y fortaleciendo el control público. A través de él, la sociedad puede determinar que los estudiantes de una determinada profesión reciban una educación de alta calidad, lo que les permitirá mejorar su calidad de vida y contribuir al desarrollo del país. Ayuda a identificar fortalezas y debilidades, permitiendo así que las ocupaciones optimicen el uso de sus recursos mediante la formulación de planes de mejora para fortalecer las primeras $y$ reducir las segundas. Las universidades pueden aprovechar los incentivos y ventajas comparativas que el Estado peruano está obligado a establecer para las ocupaciones reconocidas y sus egresados. Las carreras profesionales y sus egresados pueden obtener las recompensas y ventajas competitivas del país peruano. Otorga mayor prestigio a profesiones, universidades, estudiantes, docentes y egresados.

En la UPN, manifestaron que están comprometidos con la calidad de la educación y la organización, buscando la satisfacción del estudiante como orientación, brindando servicios educativos enfocados en el logro de capacidades, y vinculando el trabajo académico con el desarrollo de actividades de investigación, proyección social y promoción universitaria. Están dispuestos a invertir recursos para lograr una excelencia académica e institucional confiable y dependen de los sistemas de gestión de la calidad para lograr la mejora continua. Considera la calidad como parte de su cultura organizacional, y el CEO y el director firmaron los siguientes objetivos de calidad en su sitio web: asegurar la excelencia académica y organizacional. Consolidar y mejorar el sistema de servicio para asegurar la satisfacción del cliente. Implementar y desarrollar sistemas de investigación aplicada para adaptarse a las realidades nacionales, proyecciones sociales y vinculación con el proceso docente. Sobre la base del desarrollo docente y el apoyo a los estudiantes, fortalecer el aprendizaje orientado y fomentar la formación integral de los estudiantes. Licencias y certificaciones para todas las ocupaciones e instituciones enteras. Asegurar la 
estandarización e innovación curricular. Fomentar y promover una cultura de autoevaluación y mejora continua en toda la comunidad universitaria UPN. Atraer y retener talentos adecuados y desarrollarlos permanentemente. Implementar y desarrollar un sistema de contacto permanente con los grupos de interés de la organización.

La misma autoridad acordó definir los objetivos anterioresatravésdelassiguientespolíticasdecalidad: Calidad educativa y organizacional, orientada a buscar la satisfacción de nuestros estudiantes, empleadores y toda la comunidad, brindando servicios educativos enfocados al logro, capacidad y centrado en el estudiante; compaginando el trabajo académico con el desarrollo de actividades de investigación está vinculado a la proyección social. Cumplir con los requisitos legales vigentes y demás requisitos que apliquen a los servicios suscritos por la universidad. Mejorar continuamente nuestros procesos académicos y administrativos para lograr la excelencia académica e institucional.

Para cumplir con esta política la UPN cuenta con el vicerrectorado de Calidad Educativa y la Dirección de Planificación y Mejora Continua, que se encargan de consolidar el sostenimiento y desarrollo del Sistema de Gestión de Calidad de la Universidad.

\section{Expectativas del desarrollo docente}

Las universidades requieren ofrecer una educación de calidad, por ello se define enseñanza de calidad como la que consigue alcanzar las metas de enseñanza, busca que los estudiantes logren un pensamiento crítico, sean creativos y desarrollen habilidades cognoscitivas complejas. (Guzmán, 2011). La Teoría de Expectativas de Vroom, y la obra de Muchinsky (1994), refiere que este modelo se originó en 1930, y en ese entonces no se hacía referencia a la motivación laboral, fue Vroom (1964) quien catapultó la teoría de la expectativa dentro del área de la investigación sobre la motivación.

Asume que todos tomarán decisiones racionales y dedicarán su energía a actividades que brinden rendimientos ideales para el trabajo que desean, dependiendo de su desempeño. UTP (2019) confirmó que una tarea tan importante como la docencia universitaria requiere que el mismo docente se ocupe de los conceptos modernos de la asignatura dela que es responsable, y tiene una amplia experiencia en medios, porque si solo transfieres conocimientos, no afectará los estudiantes sepan comunicarlos o hacerlos interesantes y productivos. Para ello, la docencia universitaria debe basarse en tres aspectos: excelente trabajo académico, estrategias docentes actualizadas, cumplimiento de la normativa urbanística y administrativa. La relación con los estudiantes se basa en la tolerancia y el respeto a la diversidad ideológica, manteniendo así el mejor rendimiento académico. Esperar desarrollar bien su trabajo, no solo como transmisor de información, sino también como promotor de cambio, creativo e innovador, y capaz de captar las actitudes de los estudiantes y el entorno social.

La docencia es un campo muy valioso, pero requiere de estrategias de gestión para posicionar bien su presencia en el aula. La educación se está desarrollandoyla relación maestro-alumno también se está desarrollando. Universia (2020). Por tanto, la expectativa de ser un buen docente involucra tres aspectos básicos: mantener un ambiente de confianza con los estudiantes a través de una buena comunicación, y tener un diálogo sincero y fluido 
para evitar el rechazo. Estimulación positiva ante la creatividad del tema propuesto. Abandona a los típicos maestros que enseñan justicia o creen que siempre tienes la razón. Por tanto, el modelo adoptado es estar abierto al diálogo.

Lo descrito, implica las buenas prácticas docentes, requiere definir las capacidades propias de un docente ideal y con ello una "docencia de calidad". Existen muchos autores que proponen competencias y capacidades del buen docente como son (Perrenoud, 2004; Ramsden, 2006; Zabalza, 2007, entre otros). Por ello en la UPN, una de las expectativas del docente es que sea integral o ideal, aunque en todos los ámbitos resulta complejo contar con la totalidad de dichas características, a pesar del compromiso a su labor y esfuerzos por conseguirlo. La excelencia académica se entiende como la exigencia disciplinar, la rigurosidad científica y la coherencia ética. Los planes de estudio y la plana docente se combinan en cada carrera para asegurar un pleno dominio disciplinar, y en la formación de pre y posgrado se ofrece también un enfoque multi e interdisciplinar, necesario para enfrentar los retos que, en el mundo actual, plantea la sociedad, la ciencia y la naturaleza. Todo ello demanda de los docentes un alto grado de preparación, dominio de su disciplina y apertura hacia una visión interdisciplinaria de la realidad. (Del Mastro, 2020).

Describe las expectativas del profesor y muestra la relación entre esfuerzo y ejecución en el trabajo. Esto significa lo siguiente: los resultados de la labor docente que brinda: salario, promoción y vacaciones. La percepción de los docentes de los resultados (valencia) se suele definir en términos de atractivo o satisfacción esperada. Implementación y obtención de resultados (valor de la herramienta), esta cognición existe en la mente del docente. Se refiere al grado en que el logro de un determinado resultado es una condición para el desempeño del sujeto o el desempeño laboral. El esfuerzo $y$ el rendimiento (expectativas) son como el valor de una herramienta que se clasifica como probabilidad. Los docentes tienen expectativas para su propio trabajo, luego de reflexionar sobre sus esfuerzos y desempeño, los docentes realizan algunas evaluaciones. La mejor forma de formar expectativas es mediante la retroalimentación (Janz, 1982). La cantidad de esfuerzo o estrés que una persona usa para motivarse (fuerza) predice el esfuerzo. La medida más común de esfuerzo es la evaluación subjetiva, generalmente estimación: generalmente la autoevaluación del sujeto de sus esfuerzos, el juicio de los superiores o la evaluación de los colegas.

La expectativa constituye una explicación razonable y consciente de la motivación humana, explica la razón por la que ciertos trabajos parecen crear una mayor o menor motivación. Siempre que los motivos inconscientes desvíen la conducta del camino predicho por el proceso consciente de conocimiento, la teoría de la expectativa no sería productiva (Miner, 2005) sugiere que las personas difieren en el grado en que su conducta está motivada por procesos racionales. Es decir, parecen estar más motivados por factores inconscientes (Katz y Kahn, 1981). Campbell y Pritchard (1976), han demostrado la existencia de ciertas correlaciones entre rasgos de personalidad y la teoría de la expectativa.

\section{MÉTODO}

El nivel y tipo de investigación, corresponde a un nivel aplicado, el diseño es descriptivo de tipo correlacional. El estudio se realizó en la Universidad Privada del Norte con los docentes tanto a tiempo 
parcial como a tiempo completo, de las 6 sedes que a nivel nacional tiene esta institución educativa. La UPN cuenta con una población de 1778 docentes, para el estudio se planificó trabajar con un 5\% de margen de error, la muestra quedó constituida por 465 docentes. Para recopilar datos se aplicó la técnica de encuesta, se utilizaron dos instrumentos: uno sobre la escala sobre sistema de gestión de calidad (SIGECA) y el segundo sobre escala de desarrollo docente (EXDO), validada por juicio de expertos. Para obtener los resultados se aplicó los estadígrafos: Rho de Spearman, KolmogórovSmirnov y Shapiro-Wilk. A continuación, se presentan dichos resultados entre variables: sistema de gestión de calidad y expectativas de desarrollo docentes la universidad privada.

\section{RESULTADOS Y DISCUSIÓN}

En relación al análisis del sistema de gestión de la calidad y expectativas de desarrollo en docentes, el resultado arrojado fue que presentó una correlación positiva a un .697 , lo que hizo señalar que el sistema de gestión de esta casa superior de estudios si tiene un nivel de influencia y direccionamiento de las expectativas por parte de los docentes, en este caso hacia la consolidación de una organización estratégica, flexible y consciente del entorno peruano.

En cuanto al análisis entre la calidad educativa $y$ organizacional con la dimensión personal, la correlación fue positiva a un .781, indicando que la idoneidad académica y el funcionamiento eficiente de la universidad redunda positivamente en la relación carrera-vida del docente.

Seguidamente en el análisis entre la calidad educativa y organizacional con la dimensión laboral, la correlación fue positiva a un .759, lo cual indicó que hubo idoneidad académica y el funcionamiento eficiente de la universidad redunda positivamente en el crecimiento académico e incremento de la red de contactos de los docentes.

Continuando con el análisis entre los requisitos legales con la dimensión laboral, la correlación fue positiva con un .677, indicando que la estandarización e innovación curricular y el cumplimiento de los procesos de licenciamiento y acreditación de parte de la universidad redundó positivamente en el crecimiento académico $\mathrm{e}$ incremento de la red de contactos de los docentes.

Para finalizar, en el análisis que se realizó sobre la mejora continua con la dimensión laboral, la correlación fue positiva a un .748, lo que indicó que la cultura de autoevaluación, atracción-retención del talento humano como la vinculación con grupos de interés de la universidad redundara positivamente en el crecimiento académico e incremento de la red de contactos docentes.

\section{Discusión}

Carvalho y Rabelo (2016), confeccionaron una escala de expectativas de carrera en la organización donde destacaron dos elementos importantes relacionados con el desarrollo y evidencias de validez, como elemento importante para comprender la relación de los profesionales con su trabajo y la organización, cuyos resultados indican que el instrumento del estudio presenta evidencias de validez, facilitando el análisis sobre las expectativas de carrera, por lo tanto, es comparable con el presente estudio en cuanto al análisis de la calidad educativa y organizacional ya que indica idoneidad académica y el funcionamiento efectivo de la UPN con impacto positivo en la relación entre la vida profesional. 
Para Espinosa, Barrainkua y Huandicoechea (2015), quienes investigaron sobre las expectativas de la profesión, los resultados revelan, que fue constatado el interés del profesional augurando un crecimiento importante del número de profesionales de la contabilidad acreditados. Comparable con el análisis del sistema de gestión de la calidad docente y las expectativas de desarrollo muestra que el sistema de gestión de esta institución de educación superior sí tiene un cierto grado de influencia y dirección esperada en los docentes. En este caso, se trata de consolidar la organización estratégica, ser flexible y comprender la Ambiente peruano.

\section{CONCLUSIONES}

Al analizar las expectativas del sistema de gestión de la calidad y el sistema de gestión de la institución de educación superior, este si tuvo cierto grado de influencia y dirección en las expectativas de los docentes, en este caso, es necesario consolidar la organización estratégica, ser flexible y comprender el entorno peruano. La idoneidad académica y el funcionamiento eficiente de la universidad inciden positivamente en la relación de vida profesional de los docentes. La estandarización e innovación del plan de estudios, así como el cumplimiento de la universidad con los procedimientos de licencia y certificación, han tenido un impacto positivo en el desarrollo académico y el crecimiento de las redes de contacto con los docentes. La cultura de la autoevaluación, la atracción y retención de talentos y la conexión con los grupos de interés universitarios promoverá activamente el crecimiento académico y aumentará la red de contactos docentes. El desarrollo de los docentes muestra que el sistema de gestión de esta institución de educación superior sí ha tenido cierto grado de influencia y dirección esperada en los docentes, en este caso, consolidar la organización estratégica, flexible y amigable con el medio ambiente del Perú.

REFERENCIAS

Campbell, J. P., y Pritchard R. D. (1976). Motivational theory in industrial and organizational psychology. En M.D. Dunnette (Ed.), Handbook of Industrial and Organizacional psychology (pp. 63-130).

Carvalho de Vasconcellos, Vinicius y Rabelo Neiva, Elaine (2017). Escala de Expectativas de futuro organizacional: desenvolvimiento y evidencias de validade. REAd.Rev electron. Adm (Porto Alegre) 2017, vol.23, n.2, pp.5882. ISSN 1980-4164. http://www.scielo.br/ scielo.php?script $=$ sci_arttext \&pid $=$ S1413$23112017000200058 \& \operatorname{lng}=\mathrm{en} \& \mathrm{nrm}=\mathrm{iso} \& \operatorname{tn} \mathrm{ln}=$ $\mathrm{pt}$

Ceplan. (2010). Plan Bicentenario 2021. http:// www.ceplan.gob.pe/sites/default/files/plan_ bicentenario/PLAN_BICENTENARIO_ CEPLAN.pdf

Del Mastro, C. (2020). Las "buenas prácticas" del docente universitario en la PUCP. http://files.pucp.edu.pe/departamento/ educacion $/ 2020 / 02 / 11154023 /$ cristina-delmastro-las-buenas-practicas-del-docenteuniversitario-en-la-pucp.pdf

Espinosa-Pike, Marcela; Barrainkua Aroztegi, Itsaso y Huandicoechea Arondo, Miren Lorea. (2015). País Vasco. España.

Guzmán, J. (2011). La calidad de la enseñanza en educación superior ¿Qué es una buena enseñanza en este nivel educativo? Perfiles educativos, 33(spe), 129-141. http://www. scielo.org. $\mathrm{mx} / \mathrm{scielo}$.php? script $=\mathrm{sci}$ arttext\&pid=S0185-26982011000500012\&lng= es\&tlng=es.

International Organization for Standarization (2018). Norma ISO 21001:2018 para las organizaciones educativas. Global STD. Recuperado de: https://www.globalstd. com/ networks/blog/iso-21001-2018-para- 
lasorganizaciones-educativas

Janz, T. (1982). Initial comparisons of patterned behavior description interviews versus unstructured interviews. Journal of Applied Psychology, 67, 577-580.

Katz y Kahn (1981). Psicología social de las organizaciones. Ed. Trillas.

Miner, J. (2005). Organizational behavior 1. Essential theories of motivation and leadership.

Muchinsky, P. (1994). Psicología aplicada al trabajo. Desclée de Brouwer.

Perrenoud, P. (2004) Diez nuevas competencias para enseñar. Barcelona: Graó

Ramsden. P. (2006). Learning to Teach in Higher Education. (Segunda edición). Routledge

Sistema Nacional de Evaluación, Acreditación y Certificación de la Calidad Educativa, SINEACE (2017). Modelo de Acreditación para Institucional. Lima: SINEACE.
Sistema Nacional de Evaluación, Acreditación y Certificación de la Calidad Educativa (2018). Acreditación. Lima: Ministerio de Educación. https://www. sineace.gob.pe/acreditacion/

Universia (2020). ¿Cómo ser un mejor docente ante las expectativas de tus alumnos?https://orientacion.universia. edu.pe/infodetail/orientacion/consejos/ como-ser-un-mejor-docente-ante-lasexpectativas-de-tus-alumnos- $-5791 . \mathrm{html}$

UTP (2019). 10 características de un buen docente universitario. Universidad Tecnológica del Perú. Escuela de Posgrado

Vroom, V.H. (1964). Work and Motivation, John Wiley and Sons.

Zabalza, M. (2007). Competencias docentes del profesorado universitario. Calidad y desarrollo profesional. Editorial Narcea 